\title{
Facial Expression Recognition Model Based on Computer Vision
}

\author{
Chen Chao ${ }^{1}$ and Huang Linlin ${ }^{2}$ \\ ${ }^{1}$ Department of Electronic and Information Engineering, Beijing Jiaotong \\ University, Beijing, 100044, China \\ ${ }^{2}$ Department of Electronic and Information Engineering, Beijing Jiaotong \\ University, Beijing, 100044, China \\ 332124596@qq.com
}

\begin{abstract}
A new facial feature position self-calibration method based on active computer vision is proposed in this paper to realize facial expression recognition. Compared with traditional method, the proposed method based on the extension focus thought only needs four linearly independent translational movements, one real rotational movement and one virtual rotational movement rather than the calibration reference object to realize the linear solutions orderly for internal reference matrix of camera, hand-eye relationship and feature point target depth. The experiment result shows that the proposed method is feasible and effective and the measurement errors of two-dimensional and threedimensional feature points can be below $0.40 \mathrm{~mm}$, thus able to meet the industrial accuracy requirements.
\end{abstract}

Keywords: Facial expression recognition; Feature point location; Active vision; Extension focus

\section{Introduction}

As one of the most important methods for realizing robot intelligence, vision measurement and control can significantly improve the efficiency and the accuracy of robot operations, thus currently widely applied to the industrial production, especially to such fields as measurement, capture and welding. In practical application, camera is usually fixed at the end-effector of the mechanical arm of the robot so as to move along with the movement of the end-effector, thus to form a robot based hand-eye system. During robot based production operation, it is necessary to know the position of the endeffector of the mechanical arm relatively to the target object, wherein the position information of the target object can be obtained through the feature position selfcalibration and the key for information collection is to determine the relative position relationship between the end coordinate system of the mechanical arm and the coordinate system of the camera. Based on the extension focus method, a new facial expression recognition method based on active vision is proposed in this paper. The proposed method only needs two feature points rather than any calibration reference object to orderly realize the linear solutions for such information as feature position relationship rotation matrix, internal reference matrix of camera, feature point target depth and feature position relationship translation vector through accurately controlling the camera to make four linearly independent translational movements and one rotational movement. 


\section{Calibration Principle}

\subsection{Internal Reference Matrix of Camera}

Camera imaging system principle is as shown in Figure 1, wherein the imaging point coordinates of feature point $\mathrm{M}$ respectively in $\mathrm{CCD}$ imaging plane $\mathrm{O}$-uv, imaging plane $\mathrm{O}_{\mathrm{N}}-\mathrm{X}_{\mathrm{N}} \mathrm{Y}_{\mathrm{N}}$ and camera coordinate system $\mathrm{O}_{\mathrm{C}}-\mathrm{X}_{\mathrm{C}} \mathrm{Y}_{\mathrm{C}} \mathrm{Z}_{\mathrm{C}}$ are $M_{U v}(u, v), M_{N}\left(x_{n}, y_{n}\right)$ and $M_{C}\left(x_{c}\right.$, $\left.y_{c}, z_{c}\right)$.

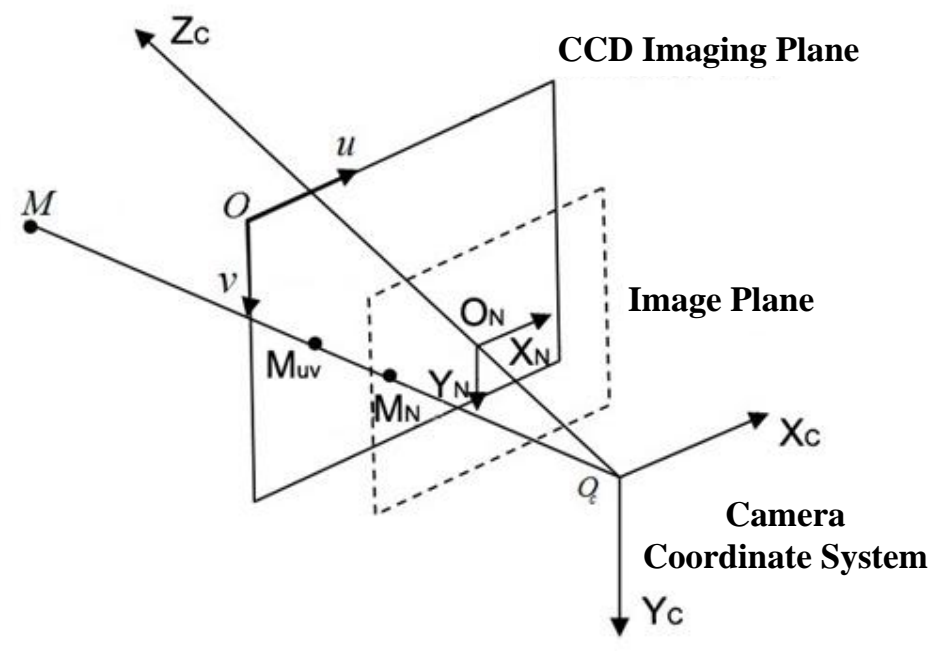

Figure 1. Camera Imaging System Principle Diagram

$K=\left[\begin{array}{ccc}\alpha & \gamma & u_{0} \\ 0 & \beta & v_{0} \\ 0 & 0 & 1\end{array}\right]$

In Formula (1), $\mathrm{K}$ is assumed as the model for the five parameters of the internal reference matrix of the camera, wherein $\alpha$ represents the scale factor of $\mathrm{x}$-axis of the image plane, $\beta$ represents the scale factor of y-axis of the image plane, $\gamma$ represents the distortion factor, and $\left(u_{0}, v_{0}\right)$ represents the origin of CCD imaging plane. The coordinate transformation relationship in the camera imaging system is as follows:

$$
\begin{gathered}
{\left[\begin{array}{c}
x_{n} \\
y_{n} \\
1
\end{array}\right]=K^{-1}\left[\begin{array}{c}
u \\
v \\
1
\end{array}\right]} \\
{\left[\begin{array}{c}
x_{c} \\
y_{c} \\
z_{c}
\end{array}\right]=z_{c}\left[\begin{array}{c}
x_{n} \\
y_{n} \\
1
\end{array}\right]}
\end{gathered}
$$

\subsection{Coordinate Relationship Transforamtion}

The active vision based hand-eye system is as shown in Figure 2, wherein the coordinates of feature point $\mathrm{M}$ in World Coordinate System $\mathrm{O}_{\mathrm{w}}-\mathrm{X}_{\mathrm{W}} \mathrm{Y}_{\mathrm{w}} \mathrm{Z}_{\mathrm{w}}$, mechanical arm end coordinate system $\mathrm{O}_{\mathrm{H}}-\mathrm{X}_{\mathrm{H}} \mathrm{Y}_{\mathrm{H}} \mathrm{Z}_{\mathrm{H}}$ and camera coordinate system $\mathrm{O}_{\mathrm{C}}-\mathrm{X}_{\mathrm{C}} \mathrm{Y}_{\mathrm{C}} \mathrm{Z}_{\mathrm{C}}$ are respectively $M_{w}\left(x_{w}, y_{w}, z_{w}\right), M_{H}\left(x_{h}, y_{h}, z_{h}\right)$ and $M_{C}\left(x_{c}, y_{c}, z_{c}\right)$. 


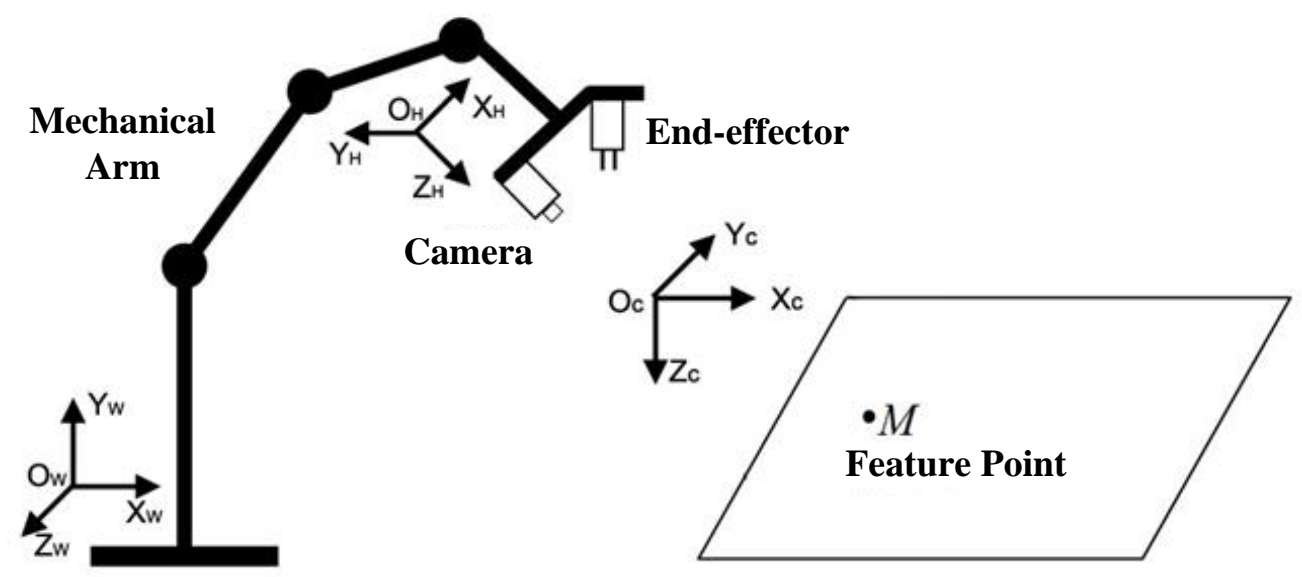

Figure 2. Active Vision Based Hand-Eye System Principle Diagram

The coordinate transformation relationship in the hand-eye system is as follows: ${ }_{C}^{H} R$ and ${ }_{C}^{H} t$ respectively represent the rotation part and the translation part from the camera coordinate system to the mechanical arm end coordinate system, ${ }_{H}^{W} R$ and ${ }_{H}^{W} t$ respectively represent the rotation part and the translation part from the mechanical arm end coordinate system to the World Coordinate System. Relevant coordinate transformation is as follows:

$$
\begin{aligned}
& {\left[\begin{array}{l}
x_{h} \\
y_{h} \\
z_{h}
\end{array}\right]={ }_{C}^{H} R\left[\begin{array}{l}
x_{c} \\
y_{c} \\
z_{c}
\end{array}\right]+{ }_{C}^{H} t} \\
& {\left[\begin{array}{l}
x_{w} \\
y_{w} \\
z_{w}
\end{array}\right]={ }_{H}^{W} R\left[\begin{array}{l}
x_{h} \\
y_{h} \\
z_{h}
\end{array}\right]+{ }_{H}^{W} t}
\end{aligned}
$$

The coordinate transformation relationship in the hand-eye system is obtained according to Formulae (1) $\sim(5)$, wherein $\varepsilon$ represents the depth value of the feature point in the camera coordinate system.

$M_{W}=\xi_{H}^{W} R_{C}^{H} R K^{-1}\left[\begin{array}{l}u \\ v \\ 1\end{array}\right]+{ }_{H}^{W} R_{C}^{H} t+{ }_{H}^{W} t$

\subsection{Extebsuib Focus}

According to the property of the extension focus [2], the corresponding connection line of any feature point before and after camera translation passes through the extension focus, so two or more feature points in the scene can be adopted to find the extension focus. 


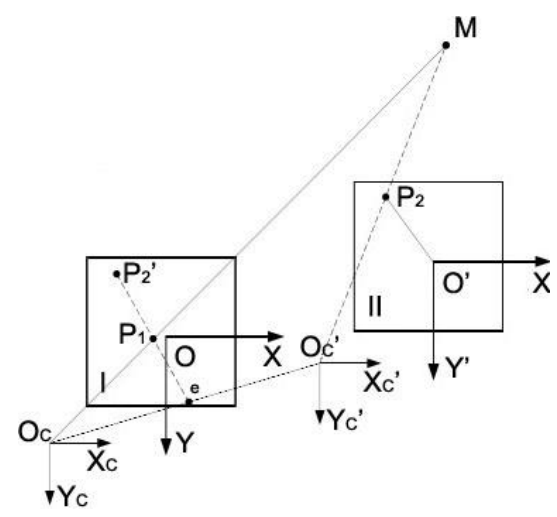

Figure 3. Extension Focus Principle Diagram

As shown in Figure 3, images I and II are the two images after camera translation, $\mathrm{P}_{1}$ and $\mathrm{P}_{2}$ are the positions of feature point $\mathrm{M}$ in the image before and after translation. In the translational movement of the camera, straight lines $\mathrm{O}_{\mathrm{C}} \mathrm{O}$ and $\mathrm{O}_{\mathrm{C}}{ }^{\prime} \mathrm{O}^{\prime}$ are parallel to each other, and straight lines $\mathrm{P}_{2}{ }^{\prime} \mathrm{O}$ and $\mathrm{P}_{2} \mathrm{O}^{\prime}$ are also parallel to each other. $\mathrm{P}_{2}$ ' is the corresponding point of $\mathrm{P}_{2}$ in image $\mathrm{I}$, connection lines $\mathrm{P}_{2}{ }^{\prime} \mathrm{P}_{1}$ and $\mathrm{O}_{\mathrm{C}} \mathrm{O}_{\mathrm{C}}{ }^{\prime}$ of the corresponding points intersect at extension focus e.

The coordinate of extension focus e in the camera coordinate system can be obtained according to Formulae (2) and (3).

$\left[\begin{array}{c}x_{c e} \\ y_{c e} \\ z_{c e}\end{array}\right]=z_{c e} K^{-1}\left[\begin{array}{c}u_{e} \\ v_{e} \\ 1\end{array}\right]=\overrightarrow{O_{c} e}=k_{1} \vec{c}$

$\overrightarrow{O_{c} O_{c}}=k \vec{c}$

$\vec{c}=\overrightarrow{O_{c} e} /\left|\overrightarrow{O_{c} e}\right|$ represents the unit vector of the translational movement of the camera, and $k>k_{1}>0$ is true.

\section{Calibration of Hand-Eye Relationship Rotation Matrix}

The camera is controlled to make one translational movement. If $\mathrm{X}_{\mathrm{H} 1}$ and $\mathrm{X}_{\mathrm{H} 2}$ are assumed to respectively represent the coordinates of feature point $\mathrm{M}$ before and after translation in the mechanical arm end coordinate system, $\mathrm{X}_{\mathrm{C} 1}$ and $\mathrm{X}_{\mathrm{C} 2}$ are assumed to respectively represent the coordinates of feature point $\mathrm{M}$ before and after translation in the camera coordinate system, then the following formula can be obtained:

$X_{H 1}={ }_{C}^{H} R X_{C 1}+{ }_{C}^{H} t$

$X_{H 2}={ }_{C}^{H} R X_{C 2}+{ }_{C}^{H} t$

The following formula can be obtained according to Formulae (9) (10):

$X_{H 2}-X_{H 1}={ }_{C}^{H} R\left(X_{C 2}-X_{C 1}\right)=k \vec{b}$

The following formula can be obtained according to Formulae (11) and (8):

$\vec{b}={ }_{c}^{H} R \vec{c}$

As shown in Formula (12), the mechanical arm is controlled to make three linearly independent translational movements to obtain the following formula:

$B={ }_{C}^{H} R C$ 
In Formula (13), $B=\left(\overrightarrow{b_{1}}, \overrightarrow{b_{2}}, \overrightarrow{b_{3}}\right)$ and $C=\left(\overrightarrow{c_{1}}, \overrightarrow{c_{2}}, \overrightarrow{c_{3}}\right)$. The following hand-eye relationship rotation matrix can be obtained:

${ }_{C}^{H} R=B C^{-1}$

Formula (14) indicates that the hand-eye relationship rotation matrix can be calibrated as an orthogonal matrix through accurately controlling the camera to make three linearly independent translational movements.

\section{Calibration of Internal Parameter Matrix of Camera}

The following formula can be obtained according to Formulae (7) and (12):

$k_{1} \vec{b}=k_{1 C}^{H} R \vec{c}={ }_{C}^{H} R \overrightarrow{O_{c} e}={ }_{C}^{H} R z_{c e} K^{-1}\left[\begin{array}{c}u_{e} \\ v_{e} \\ 1\end{array}\right]$

Formula (15) is equivalent to the following formula:

$k_{1} K_{H}^{C} R \vec{b}=z_{c e}\left[\begin{array}{c}u_{e} \\ v_{e} \\ 1\end{array}\right]$

The following equation is assumed:

$A=K_{H}^{C} R=\left[\begin{array}{lll}a_{11} & a_{12} & a_{13} \\ a_{21} & a_{22} & a_{23} \\ a_{31} & a_{32} & a_{33}\end{array}\right]$

The following formula can be obtained according to Formulae (16) and (17):

$k_{1} A\left[\begin{array}{l}b_{i 1} \\ b_{i 2} \\ b_{i 3}\end{array}\right]=z_{c e}\left[\begin{array}{c}u_{i} \\ v_{i} \\ 1\end{array}\right]$

Namely:

$\left\{\begin{array}{l}a_{11} b_{i 1}+a_{12} b_{i 2}+a_{13} b_{i 3}=u_{i}\left(a_{31} b_{i 1}+a_{32} b_{i 2}+a_{33} b_{i 3}\right) \\ a_{21} b_{i 1}+a_{22} b_{i 2}+a_{23} b_{i 3}=v_{i}\left(a_{31} b_{i 1}+a_{32} b_{i 2}+a_{33} b_{i 3}\right)\end{array}\right.$

As shown in Formulae (18), and (19), another matrix A' is assumed to have the same elements as matrix A except $a_{33}=1$, and the camera is controlled to make four linearly independent translational movements, thus to obtain the unique solution through the least square method. In order to determine $a_{33}$, the following formula is obtained through Formula (17):

$A=a_{33} A^{\prime}=a_{33}\left[\begin{array}{l}\overrightarrow{a_{1}} \\ \overrightarrow{a_{2}} \\ \overrightarrow{a_{3}}\end{array}\right]=K_{H}^{C} R=\left[\begin{array}{ccc}\alpha & \gamma & u_{0} \\ 0 & \beta & v_{0} \\ 0 & 0 & 1\end{array}\right]\left[\begin{array}{l}\overrightarrow{r_{1}} \\ \overrightarrow{r_{2}} \\ \overrightarrow{r_{3}}\end{array}\right]$

${ }_{H}^{C} R$ is an orthogonal matrix, namely $\left({ }_{H}^{C} R\right)^{-1}=\left({ }_{H}^{C} R\right)^{T}$, so the following formula can be obtained:

$a_{33}=\left|\overrightarrow{r_{3}}\right| /\left|\overrightarrow{a_{3}}\right|=1 /\left|\overrightarrow{a_{3}}\right|$

After $a_{33}$ is determined, matrix A can be found according to Formula (20) in order to calibrate the internal parameter matrix of the camera. 


\section{Calibration of Feature Point target Depth}

As shown in Figure 1, the camera is controlled to make one translational movement, wherein ( $\mathrm{u} 1, \mathrm{v} 1)$ and $(\mathrm{u} 2, \mathrm{v} 2)$ are the coordinates of feature point $\mathrm{M}$ before and after translation in CCD imaging plane, $(\mathrm{x} 1, \mathrm{y} 1)$ and $(\mathrm{x} 2, \mathrm{y} 2)$ are the coordinates of feature point $\mathrm{M}$ before and after translation in the image plane, $\mathrm{MC1}(\mathrm{xc} 1, \mathrm{yc} 1, \mathrm{zc} 1)$ and $\mathrm{MC} 2(\mathrm{xc} 2, \mathrm{yc} 2, \mathrm{zc} 2)$ are the coordinates of feature point $\mathrm{M}$ before and after translation in the camera coordinate system. The following formula can be obtained according to formulae (2), (3) and (8):

$$
\begin{aligned}
& \left\{\left[\begin{array}{l}
x_{c 1} \\
y_{c 1} \\
z_{c 1}
\end{array}\right]=z_{1} K^{-1}\left[\begin{array}{c}
u_{1} \\
v_{1} \\
1
\end{array}\right]\right. \\
& {\left[\begin{array}{l}
x_{c 2} \\
y_{c 2} \\
z_{c 2}
\end{array}\right]=z_{2} K^{-1}\left[\begin{array}{c}
u_{2} \\
v_{2} \\
1
\end{array}\right]} \\
& \overrightarrow{M_{C 1} M_{c 2}}=k \vec{c}
\end{aligned}
$$

Formula (22) is put into Formula (23) to obtain the following formula according to Formula (1):

$z_{c 2}\left[\begin{array}{c}u_{2} \\ v_{2} \\ 1\end{array}\right]-z_{c 1}\left[\begin{array}{c}u_{1} \\ v_{1} \\ 1\end{array}\right]=k K \vec{c}=k\left[\begin{array}{ccc}\alpha & \gamma & u_{0} \\ 0 & \beta & v_{0} \\ 0 & 0 & 1\end{array}\right]\left[\begin{array}{c}c_{1} \\ c_{2} \\ c_{3}\end{array}\right]$

Namely:

$$
\left\{\begin{array}{c}
z_{c 2} u_{2}-z_{c 1} u_{1}=k\left(\alpha c_{1}+\gamma c_{2}+u_{0} c_{3}\right) \\
z_{c 2} v_{2}-z_{c 1} v_{1}=k\left(\beta c_{2}+v_{0} c_{3}\right) \\
z_{c 2}-z_{c 1}=k c_{3}
\end{array}\right.
$$

Formula (25) is simplified to obtain:

$$
\left\{\begin{array}{c}
z_{c 1}\left(u_{2}-u_{1}\right)=k\left(\alpha c_{1}+\gamma c_{2}+u_{0} c_{3}-c_{3} u_{2}\right) \\
z_{c 1}\left(v_{2}-v_{1}\right)=k\left(\beta c_{2}+v_{0} c_{3}-c_{3} v_{2}\right)
\end{array}\right.
$$

When $\mathrm{u} 2-\mathrm{u} 1 \neq 0$ is true, the solution of Formula (26) is as follows:

$z_{c 1}=\frac{k\left(\alpha c_{1}+\gamma c_{2}+u_{0} c_{3}-c_{3} u_{2}\right)}{u_{2}-u_{1}}$

Or, when $v 2-v 1 \neq 0$ is true, the solution is as follows:

$z_{c 1}=\frac{k\left(\beta c_{2}+v_{0} c_{3}-c_{3} v_{2}\right)}{v_{2}-v_{1}}$

Similarly, $z_{c 2}$ can be evaluated through Formula (26). Meanwhile, Formulae (26) (28) also indicate that the target depth information of the feature point before and after translation can be obtained through controlling the camera to make one translational movement. 


\section{Calibration of Facial Feature Relationship Translation Vector}
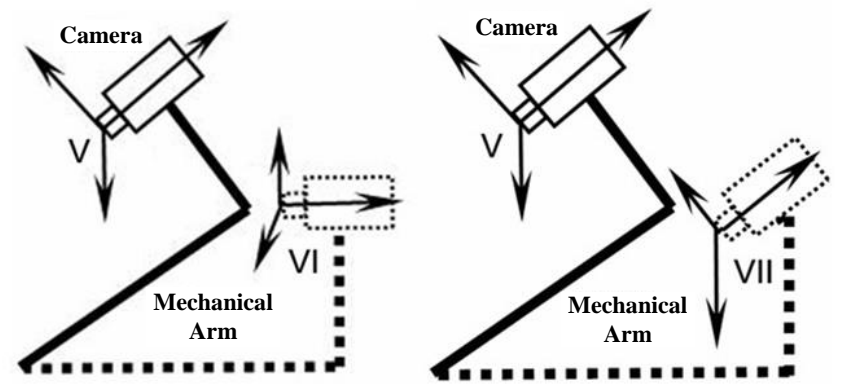

Figure 4. Comparison Diagram for System Posture Before and After Real Rotation and Virtual Rotation

As shown in Figure 4, after four linearly independent translational movements, the camera is controlled to make one real rotational movement (Posture $\mathrm{V} \rightarrow$ Posture VI) and then make one virtual rotational movement (Posture VI -> Posture VII). The corresponding numerical axes of the camera coordinate system after virtual rotation and the camera coordinate system before real rotation are parallel to each other in the same direction.

$\mathrm{X}_{\mathrm{H} 5}$ and $\mathrm{X}_{\mathrm{H} 6}$ are the coordinates of feature point $\mathrm{M}$ before and after real rotation in the mechanical arm end coordinate system, $\mathrm{X}_{\mathrm{C} 5}$ and $\mathrm{X}_{\mathrm{C} 6}$ are the coordinates of feature point $\mathrm{M}$ before and after real rotation in the camera coordinate system. Then, the following formula can be obtained:

$\left\{\begin{array}{c}X_{C 5}={ }_{H}^{C} R X_{H 5}+{ }_{H}^{C} t \\ X_{C 6}={ }_{H}^{C} R X_{H 6}+{ }_{H}^{C} t \\ X_{H 6}=R_{H} X_{H 5}\end{array}\right.$

The following formula can be obtained according to Formula (29):

$X_{C 6}={ }_{H}^{C} R R_{H C}^{H} R X_{C 5}+\left(E-{ }_{H}^{C} R R_{H}{ }_{C}^{H} R\right){ }_{H}^{C} t$

Where ${ }_{H}^{C} R$ is an orthogonal matrix and has been solved through controlling the camera to make three translational movements in above paragraph, $R_{H}$ represents the rotation matrix of the mechanical arm end coordinate system before and after the mechanical arm rotation and can be obtained through the mechanical arm controller.

As shown in Figure 4, after virtual rotation, the coordinates of feature point $\mathrm{M}$ in the mechanical arm end coordinate system and the camera coordinate system are respectively $\mathrm{X}_{\mathrm{H} 7}$ and $\mathrm{X}_{\mathrm{C} 7}$. Then, the following formula can be obtained:

$X_{C 7}={ }_{H}^{C} R R_{H}{ }_{C}^{-1 H} R X_{C 6}$

The following formula can be obtained according to Formulae (30) and (31):

$X_{C 7}=X_{C 5}+\left({ }_{H}^{C} R R_{H}{ }^{-1}{ }_{C}^{H} R-E\right){ }_{H}^{C} t$

Formula (32) indicates that the coordinates of feature point $\mathrm{M}$ in the camera coordinate system respectively after one real rotation and one virtual rotation are $X_{C 5}\left(x_{c 5}, y_{c 5}, z_{c 5}\right)$ and $X_{C 7}\left(x_{c 7}, y_{c 7}, z_{c 7}\right)$, and such process can be regarded as the coordinate transformation before and after one translational movement.

The following formula can be obtained according to Formulae (2), (3) and (31): 
$z_{c 7}\left[\begin{array}{c}u_{7} \\ v_{7} \\ 1\end{array}\right]=z_{c 6} K_{H}^{C} R R_{H}{ }^{-1 H} R K^{-1}\left[\begin{array}{c}u_{6} \\ v_{6} \\ 1\end{array}\right]$

The following equations are assumed:

$a=z_{c 7} / z_{c 6}$

$D=K_{H}^{C} R R_{H}{ }_{C}^{-1} R K^{-1}=\left[\begin{array}{lll}d_{11} & d_{12} & d_{13} \\ d_{21} & d_{22} & d_{23} \\ d_{31} & d_{32} & d_{33}\end{array}\right]$

Formulae (34) and (35) are put into Formula (33) to obtain the following formula:

$a\left[\begin{array}{c}u_{7} \\ v_{7} \\ 1\end{array}\right]=\left[\begin{array}{lll}d_{11} & d_{12} & d_{13} \\ d_{21} & d_{22} & d_{23} \\ d_{31} & d_{32} & d_{33}\end{array}\right]\left[\begin{array}{c}u_{6} \\ v_{6} \\ 1\end{array}\right]$

Formula (36) is expanded to obtain:

$\left\{\begin{array}{l}u_{7}=\frac{d_{11} u_{6}+d_{12} v_{6}+d_{13}}{d_{31} u_{6}+d_{32} v_{6}+d_{33}} \\ v_{7}=\frac{d_{21} u_{6}+d_{22} v_{6}+d_{23}}{d_{31} u_{6}+d_{32} v_{6}+d_{33}}\end{array}\right.$

In Formula (37), nine elements of matrix $\mathrm{D}$ are known, $\left(u_{6}, v_{6}\right)$ is the coordinate of feature point $M$ after the real rotation of the mechanical arm in CCD imaging plane. Accordingly, the coordinate of feature point $M$ after virtual rotation of the mechanical arm in CCD imaging plane is evaluated as $\left(u_{7}, v_{7}\right)$ and further the coordinate of feature point $\mathrm{M}$ after virtual rotation in the camera coordinate system is evaluated as $X_{C 7}\left(x_{c 7}, y_{c 7}, z_{c 7}\right)$.

The following formula can be obtained according to Formulae (2), (3) and (8):

$$
\begin{gathered}
\left\{\begin{array}{l}
{\left[\begin{array}{l}
x_{c 5} \\
y_{c 5} \\
z_{c 5}
\end{array}\right]=z_{c 5} K^{-1}\left[\begin{array}{l}
u_{5} \\
v_{5} \\
1
\end{array}\right]} \\
{\left[\begin{array}{l}
x_{c 7} \\
y_{c 7} \\
z_{c 7}
\end{array}\right]=z_{c 7} K^{-1}\left[\begin{array}{l}
u_{7} \\
v_{7} \\
1
\end{array}\right]}
\end{array}\right. \\
X_{c 7}-X_{c 5}=k \vec{c}
\end{gathered}
$$

Since the corresponding relationship of $\left(u_{5}, v_{5}\right)_{->}\left(u_{7}, v_{7}\right)$ is similar to one translational movement, thus unit vector $\vec{c}$ of the translational movement can be found according to the coordinates of the two points.

Formulae (1) and (38) are put into Formula (39) to obtain the following formula:

$z_{c 7}\left[\begin{array}{c}u_{7} \\ v_{7} \\ 1\end{array}\right]-z_{c 5}\left[\begin{array}{c}u_{5} \\ v_{5} \\ 1\end{array}\right]=k^{\prime}\left[\begin{array}{ccc}\alpha & \gamma & u_{0} \\ 0 & \beta & v_{0} \\ 0 & 0 & 1\end{array}\right]\left[\begin{array}{l}c_{1}^{\prime} \\ c_{2} \\ c_{3}^{\prime}\end{array}\right]$

Namely: 
$\left\{\begin{array}{c}z_{c 7} u_{7}-z_{c 5} u_{5}=k^{\prime}\left(\alpha c_{1}^{\prime}+\gamma c_{2}^{\prime}+u_{0} c_{3}^{\prime}\right) \\ z_{c 7} v_{7}-z_{c 5} v_{5}=k^{\prime}\left(\beta c_{2}^{\prime}+v_{0} c_{3}^{\prime}\right) \\ z_{c 7}-z_{c 5}=k^{\prime} c_{3}^{\prime}\end{array}\right.$

Formula (41) is simplified to obtain:

$\left\{\begin{array}{c}z_{c 5}\left(u_{7}-u_{5}\right)=k^{\prime}\left(\alpha c_{1}^{\prime}+\gamma c_{2}^{\prime}+u_{0} c_{3}^{\prime}-c_{3}^{\prime} u_{7}\right) \\ z_{c 5}\left(v_{7}-v_{5}\right)=k^{\prime}\left(\beta c_{2}^{\prime}+v_{0} c_{3}^{\prime}-c_{3}^{\prime} v_{7}\right)\end{array}\right.$

When $\alpha c_{1}^{\prime}+\gamma c_{2}^{\prime}+u_{0} c_{3}^{\prime}-c_{3}^{\prime} u_{7} \neq 0$ is true, the solution of Formula (42) is as follows:

$k^{\prime}=\frac{z_{c 5}\left(u_{7}-u_{5}\right)}{\alpha c_{1}^{\prime}+\gamma c_{2}^{\prime}+u_{0} c_{3}^{\prime}-c_{3}^{\prime} u_{7}}$

Or, when $\beta c_{2}^{\prime}+v_{0} c_{3}^{\prime}-c_{3}^{\prime} v_{7} \neq 0$ is true, the solution is as follows:

$k^{\prime}=\frac{z_{c 5}\left(v_{7}-v_{5}\right)}{\beta c_{2}^{\prime}+v_{0} c_{3}^{\prime}-c_{3}^{\prime} v_{7}}$

$k$ can be evaluated according to Formula (43) or (44), and then the following formula can be obtained according to Formulae (32) and (39):

${ }_{H}^{C} t=k^{\prime}\left({ }_{H}^{C} R R_{H}{ }_{C}^{-1 H} R-E\right)^{-1} \vec{c}$

According to Formula (45), the hand-eye relationship translation vector can be calibrated through controlling the mechanical arm to make one real rotation and one virtual rotation.

\section{Simulation Experiment}

\subsection{Simulation Environment and Data Source}

In order to verify the effectiveness of the facial recognition algorithm, Matlab 2012 is adopted for the simulation experiment through programming in the environment: Intel 4-core, 3.0 GHz CPU, 4GB RAM, Windows XP operating system, wherein the data are sourced from AR and Yale B face database. Some classic facial recognition algorithms are selected for the comparison experiment, such as PCA, RPCA and the facial recognition algorithms proposed in literatures [16-17]. Additionally, AR face database involves 126 persons and each person has 26 images, and some samples of AR face database are as shown in Figure 5.

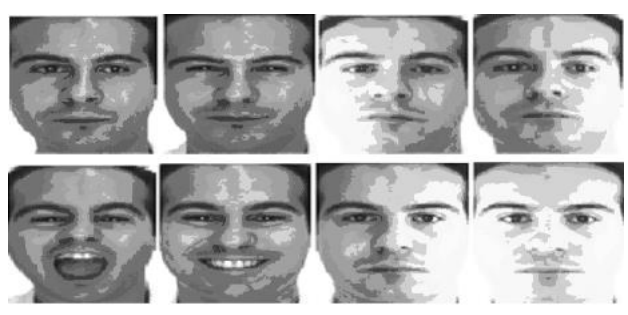

Figure 5. Faces of AR Face Database

Yale database involves 15 persons, totally 165 images under the same illumination change, expression change and background, and some samples of Yale $\mathrm{B}$ database are as shown in Figure 6. 


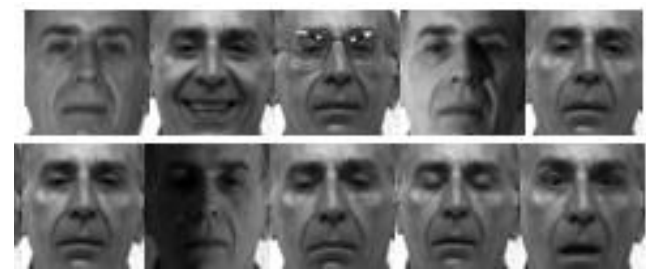

Figure 6. Faces of Yale B Face Database

\subsection{Result and Analysis}

For AR face database, 10 images of each type of faces are randomly selected as the training samples and the rest images are taken as the test samples, and the simulation results of all algorithms are as shown in Figure 7. According to Figure 7, the recognition rate of the proposed facial recognition algorithm can reach $95.14 \%$, the algorithm in literature [17] is only inferior to the proposed algorithm, with the facial recognition rate as $90.15 \%$, the facial recognition rates of the algorithm in literature [16] and RPCA algorithm are respectively $88.12 \%$ and $85.10 \%$, and PCA algorithm has the lowest recognition rate as $67.15 \%$. The above comparison result shows that the existing algorithms cannot well implement the feature extraction and the dimensionality reduction for the facial images, and the feature information redundancy is serious, thus adversely influencing the subsequent facial recognition; the proposed algorithm can well solve the problems existing in traditional feature extraction and dimensionality reduction method and obtain better facial recognition result, thus having obvious superiority.

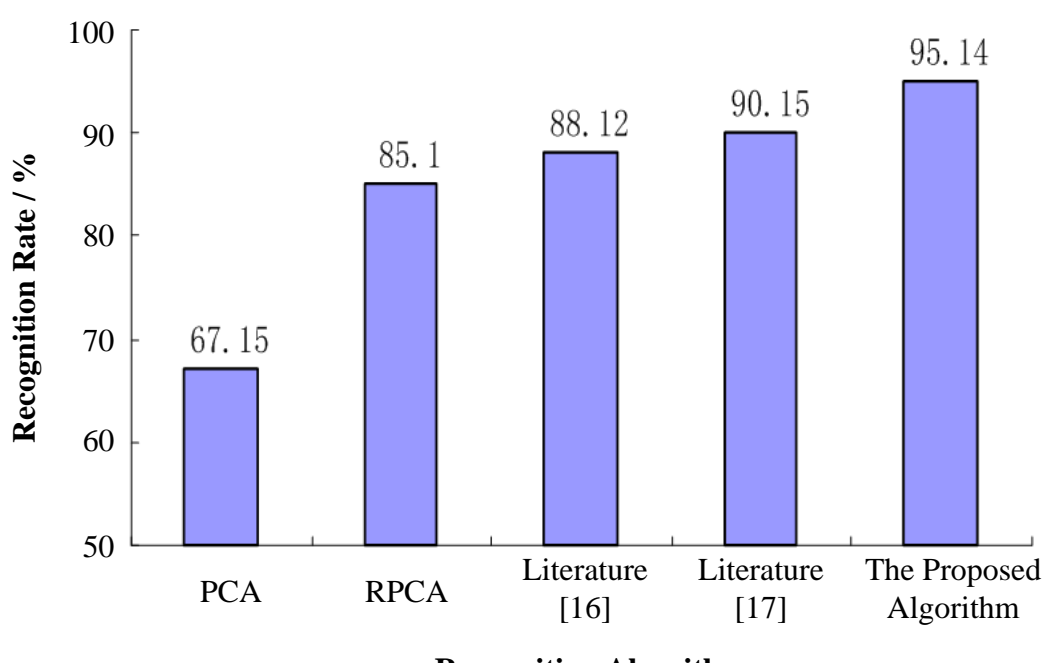

Recognition Algorithm

Figure 7. Comparison of Recognition Rates of Different Algorithms in AR Face Database

For Yale B face database, 7 images of each type of faces are randomly selected as the training samples and the rest images are taken as the test samples, and the simulation results of all algorithms are as shown in Figure 8. According to Figure 8, compared with the existing classic facial expression recognition algorithms, the proposed facial expression recognition algorithm has the highest recognition rate as $90.11 \%$, thus indicating that the proposed algorithm has better robustness to the faces under such conditions as illumination change, shadow and shielding and can obtain more ideal facial expression recognition effect. Therefore, the superiority of the proposed facial expression recognition algorithm is proven again. 


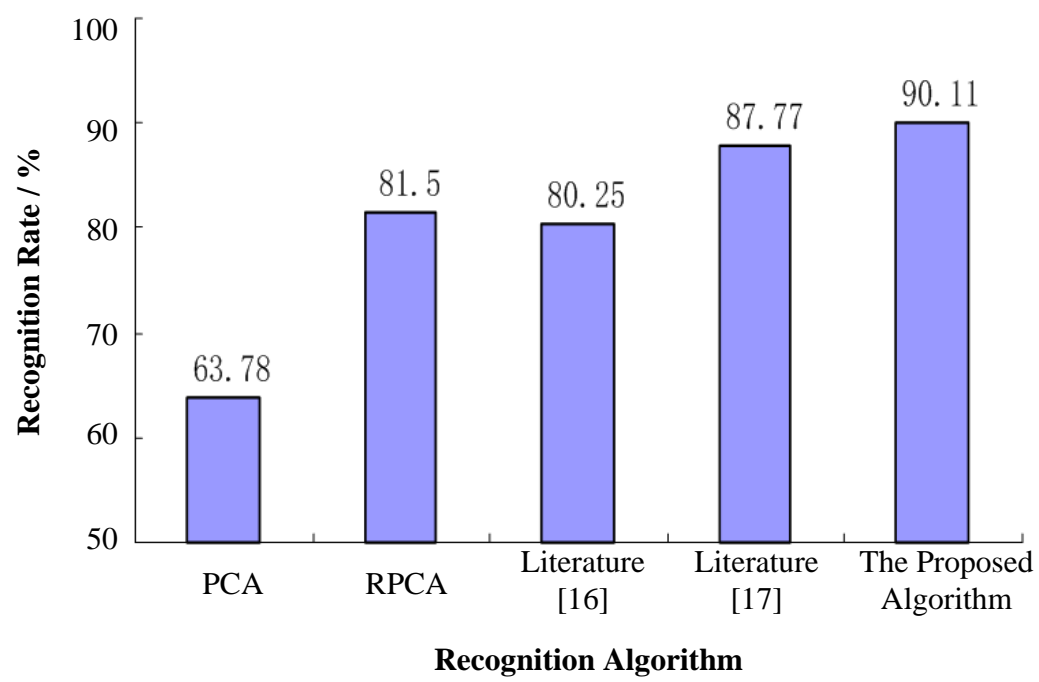

\section{Figure 8. Comparison of Recognition Rates of Different Algorithms in Yale B Face Database}

The average facial expression recognition time of all algorithms is as shown in Table 1. According to Table 1, the proposed facial expression recognition algorithm has shortest average recognition time and fastest recognition speed, thus widening the application scope of the facial expression recognition.

\section{Table 1. Comparison of Facial Expression Recognition Effects of all Algorithms}

\begin{tabular}{ccc}
\hline Algorithm & AR Faces & Yale B Faces \\
\hline PCA & 3.017 & 3.367 \\
RPCA & 2.063 & 2.143 \\
Algorithm in & 2.282 & 3.414 \\
Literature [16] & & \\
Algorithm in & 2.542 & 2.771 \\
Literature [17] & 1.296 & 1.652 \\
Proposed Algorithm & & \\
\hline
\end{tabular}

\section{References}

[1] W. Gu, Z. Lv and M. Hao, "Change detection method for remote sensing images based on an improved Markov random field", Multimedia Tools and Applications, (2015), pp. 1-16.

[2] Z. Lv, A. Tek and F. D. Silva, "Game on, science-how video game technology may help biologists tackle visualization challenges", PloS one, vol. 8, no. 3, (2013), pp. 57990.

[3] Z. Chen, W. Huang and Z. Lv, "Towards a face recognition method based on uncorrelated discriminant sparse preserving projection", Multimedia Tools and Applications, (2015), pp. 1-15.

[4] D. Jiang, X. Ying and Y. Han, "Collaborative multi-hop routing in cognitive wireless networks", Wireless Personal Communications, (2015), pp. 1-23.

[5] Z. Lv, A. Tek and F. D. Silva, "Game on, science-how video game technology may help biologists tackle visualization challenges", PloS one, vol. 8, no. 3, (2013), pp. 57990.

[6] D. Jiang, Z. Xu and Z. Lv, "A multicast delivery approach with minimum energy consumption for wireless multi-hop networks", Telecommunication Systems, (2015), pp. 1-12.

[7] C. Fu, P. Zhang and J. Jiang, "A Bayesian approach for sleep and wake classification based on dynamic time warping method", Multimedia Tools and Applications, (2015), pp. 1-20.

[8] J. Yang, S. He and Y. Lin, "Multimedia cloud transmission and storage system based on internet of things", Multimedia Tools and Applications, (2015). 
[9] C. Guo, X. Liu and M. Jin, "The research on optimization of auto supply chain network robust model under macroeconomic fluctuations", Chaos, Solitons \& Fractals, (2015).

[10] X. Li, Z. Lv and J. Hu, "XEarth: A 3D GIS Platform for managing massive city information", Computational Intelligence and Virtual Environments for Measurement Systems and Applications (CIVEMSA), 2015 IEEE International Conference on. IEEE, (2015), pp. 1-6.

[11] J. Yang, B. Chen and J. Zhou, "A Low-Power and Portable Biomedical Device for Respiratory Monitoring with a Stable Power Source”, Sensors, vol. 15, no. 8, (2015), pp. 19618-19632.

[12] G. Bao, L. Mi, Y. Geng and K. Pahlavan, "A computer vision based speed estimation technique for localiz ing the wireless capsule endoscope inside small intestine", 36th Annual International Conference of the IEEE Engineering in Medicine and Biology Society (EMBC), (2014) August.

[13] X. Song and Y. Geng, "Distributed community detection optimization algorithm for complex networks", Journal of Networks, vol. 9, no. 10, (2014) January, pp. 2758-2765

[14] D. Jiang, X. Ying and Y. Han, "Collaborative multi-hop routing in cognitive wireless networks", Wireless Personal Communications, (2015), pp. 1-23.

[15] J. Hu and Z. Gao, "Modules identification in gene positive networks of hepatocellular carcinoma using Pearson agglomerative method and Pearson cohesion coupling modularity", Journal of Applied Mathematics, vol. 2012, (2012).

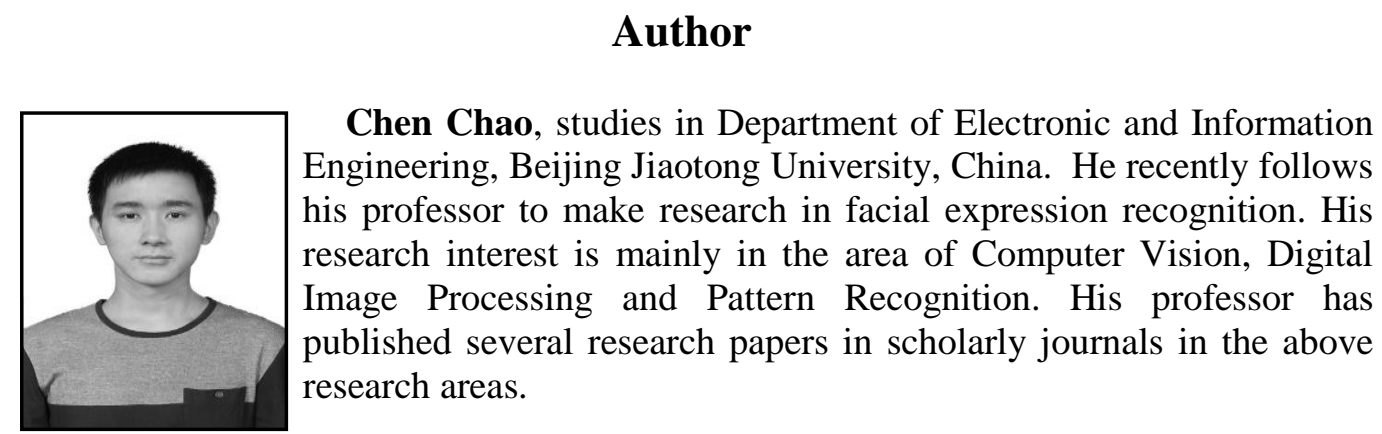

